\title{
The effects of weather on daily emergency ambulance service demand in Taipei: a comparison with Hong Kong
}

\author{
Ho Ting Wong ${ }^{1} \cdot$ Jen-Jia Lin ${ }^{2}$
}

Received: 19 September 2019 / Accepted: 2 April 2020 / Published online: 21 April 2020

(C) Springer-Verlag GmbH Austria, part of Springer Nature 2020

\begin{abstract}
Numerous studies have examined the effects of weather on emergency ambulance service (EAS) demand. Given Taipei's unique physical and social environments, empirical evidence collected from other regions may not be applicable. Collecting more information about the characteristics of vulnerable groups and the effects of weather could help the EAS managing authority in formulating cost-effective EAS policies. This study aims to look at the effects of weather on EAS demand in Taipei and to make a comparison with Hong Kong, which is also an Asian city and has a similar cultural context. The study analyzed over 370,000 EAS usage records from the Taipei City Fire Department. These records were aggregated into time series data according to patients' characteristics and then regressed on meteorological data via multivariate forward regression. The effect size differences of the variance explained by different groups of EAS users' regression models were compared. Afterward, the results of the regression analysis from Taipei were compared with those from a Hong Kong study. Elderly and critical patients in both cities showed significantly more sensitivity to weather than other patients. Further analysis showed that non-trauma cases were related to weather in Taipei. Although both cities had similar results, the Taipei study clearly showed that elderly and critical patients were more sensitive to weather than other patient subgroups. Health education programs should focus on the vulnerable groups identified in this study in order to increase their awareness and help them protect themselves before the onset of adverse weather conditions. By generating results that are directly applicable to Taipei, the formulation of inappropriate EAS policies can be prevented.
\end{abstract}

\section{Background}

Rapid urbanization in many countries is one of the many factors that has resulted in a long-term increase in global temperature and has made climate change a critical issue discussed by different parties. The general consensus is that the increasing frequency of extreme weather conditions, such as very hot days, is a product of climate change (Chapman et al. 2017; Griffiths et al. 2005). To understand further the consequences

Ho Ting Wong

frankwong@connect.hku.hk

Jen-Jia Lin

jenjia@ntu.edu.tw

1 Institute of Health Care Management, Department of Business Management, National Sun Yat-sen University, 70 Lienhai Rd., Kaohsiung 80424, Taiwan

2 Department of Geography, National Taiwan University, Taipei City, Taiwan of climate change, many studies have examined the relationship between weather and human health. Among different health outcomes, death rate (Chan et al. 2012; Goggins et al. 2013) and hospitalization rate (Chan et al. 2013) are two of the most popular research targets. Various diseases have also been linked to weather, including heart failure (Goggins and Chan 2017), asthma (Lam et al. 2016; Qiu et al. 2015), and stroke (Goggins et al. 2012). Nowadays, health records such as hospitalization and death records are commonly collected in digitalized format; this is among the main reasons why the above studies have become popular (Wong et al. 2015; Wong et al. 2016). In contrast, similar studies on the demand for emergency ambulance services (EAS) are relatively few in number, even though the number of EAS users is obviously much larger than the number of hospitalized patients and death cases. This situation is reflected in the number of journals in the category of emergency medicine and other health-related categories shown by the Clarivate Analytics' Journal Citation Reports (Clarivate Analytics 2018). Moreover, it is also believed that the effects of climate are stronger on EAS than on 
hospitalization and mortality (CCOUC 2019). As paramedics are the first health professionals to provide medical care to patients in emergency situations, EAS has become a critical part of the health service system. Knowing the effects of weather on EAS demand can help authorities to formulate health policies and also help people to protect themselves before the onset of adverse weather conditions. Some reports show that EAS demand in different countries is increasing yearly (Lai and Wong 2015; Lowthian et al. 2011). Therefore, understanding the abovementioned effects of weather can also help EAS managing authorities to cope with unexpected sudden increases in EAS demand under limited resources. For example, there are research reports that have recorded success in developing a 7-day EAS demand forecast system, using weather forecast reports and based on the identified significant meteorological predictors of EAS demand (Wong and Lai 2014). Moreover, the effects of such predictors on long-term EAS demand could also be projected to help EAS managing authorities to achieve timely responses (Lai and Wong 2015).

However, relatively few studies across the world have examined the relationship between weather and EAS demand. In the Western world, Thornes et al. (2014) analyzed 5 years (2007-2011) of EAS call-out data for Birmingham in the UK, and found that the number of EAS call-outs significantly increased during cold weather. They also found that the impact of the cold weather of December 2010 on EAS response time was almost as large as the impact of the swine flu pandemic in July 2009. Given that the increase in EAS demand has been mainly attributed to patients with certain diseases, some researchers have conducted similar studies by focusing only on these diseases. For example, Turner et al. (2013) analyzed 783,935 ambulance usage records from 2000 to 2007 for Brisbane in Australia, specifically focusing on cardiovascular patients. They found that EAS demand for cardiovascular patients was significantly increased by sustained periods of high temperature. This finding underscored the importance of installing heat warning systems that will assist with managing EAS during severe heat events. In contrast, Vencloviene et al. (2015) focused on patients with acute coronary syndrome (ACS) and included additional factors in their analysis, including relative humidity, barometric pressure, and wind speed. They concluded that, in addition to air temperature, barometric pressure and wind speed were also potential predictors of ACS.

In the Eastern world, Wong and Lai (2012) found that people with different social demographic characteristics showed different sensitivities to weather. Based on their analysis of daily EAS usage statistics in Hong Kong, they found that the elderly, poor, women, and more severe patients were relatively more vulnerable to weather. They obtained similar results from their analysis of monthly EAS demand statistics, which showed that age and socioeconomic status were closely related to vulnerable groups (Wong et al. 2017). Similar studies have also been conducted in Asia-Pacific countries such as Japan (Onozuka and Hagihara 2016; Onozuka and Hagihara 2017), Korea (Kim et al. 2012), and China (Chen et al. 2015; Yang et al. 2014). Surprisingly, the impact of adverse weather conditions on EAS demand in Taiwan has never been investigated in the literature. Although the negative impacts of extreme weather conditions on human health are significant, there is a paucity of publications related to weather effects on ambulance demand in Taiwan when searching the most popular medical journals database, "PubMed," using the search query of "(ambulance) AND (weather OR temperature) AND (Taiwan OR Taipei)." Lin (2015) has also pointed out that there is a lack of research on factors affecting Taiwan's EAS demand.

Given the differences in the climate, built environment, and lifestyle between Taiwan and Hong Kong, the findings of Wong and Lai (2012) in Hong Kong may not be applicable to Taiwan. For example, the daily temperature range in Taipei is significantly wider in both value and variation than that in Hong Kong (Table 1). Moreover, given that the distance between buildings in Taiwan is significantly further than that in Hong Kong, people in Taiwan experience longer periods of outdoor exposure when their daily activities require them to travel from building to building, therefore giving them less protection from the outdoor environment. The Taiwanese are also not in the habit of using air conditioning all the time, which is considered common practice in Hong Kong, and therefore, they also have less protection from significant changes in temperature. As the capital of Taiwan, Taipei is one of the top ten destinations in the Asia-Pacific region in terms of international overnight arrivals, with around 7.4 million international visitors in 2016 (Mastercard 2017). Therefore, studying the effects of weather on EAS demand in Taiwan is meaningful not only to local residents but also to international visitors. The Taipei city government does also recognize the negative impacts, and efforts have been made to help vulnerable groups protect themselves from adverse weather conditions. However, it is not uncommon for significant increases in deaths on cold days to be reported. In particular, during the 9-h period from 11 pm on 21 January 2019 to 8 am on 22 January 2019 , when the temperature dropped below $10{ }^{\circ} \mathrm{C}$, eleven out-of-hospital cardiac arrest cases were reported in the cities of Taipei and New Taipei. Gathering more information about vulnerable groups and ascertaining when they will be affected by weather could help EAS managing authorities in formulating cost-effective EAS management strategies and policies. Therefore, this study aims to look at the effects of weather on EAS demand in Taipei by analyzing EAS usage records. 
Table 1 The profile of average temperature and change of temperature within a day in Taipei and Hong Kong

\begin{tabular}{|c|c|c|c|c|c|c|}
\hline \multirow[t]{2}{*}{ Temperature factor $\left({ }^{\circ} \mathrm{C}\right)$} & \multicolumn{2}{|l|}{ Mean } & \multicolumn{2}{|c|}{ Maximum value } & \multicolumn{2}{|c|}{ Standard deviation } \\
\hline & Taipei & Hong Kong & Taipei & Hong Kong & Taipei & Hong Kong \\
\hline$T_{\text {avg }}$ & 23.52 & 23.46 & 32.80 & 31.20 & 5.54 & 4.91 \\
\hline$T_{\max }-T_{\min }$ & 6.42 & 4.17 & 17.00 & 11.00 & 2.81 & 1.50 \\
\hline
\end{tabular}

\section{Methods}

\subsection{Data}

\subsubsection{EAS usage records}

This study analyzed over 370,000 EAS usage records from April 2010 to December 2012, obtained from the Taipei City Fire Department. Only data up to 2012 were used in order to prevent the data period from overlapping with the 23-day Sunflower Student Movement in March 2013 and subsequent political events (Brindle 2016), which may have had significant impacts on EAS demand despite being unrelated to weather. The collected EAS usage records include five variables, including date, age, gender, triage level (level 1: critical; level 2: emergency; level 3: urgent; level 4: semi-urgent; level 5: non-urgent), and case nature (trauma or non-trauma). The case nature for each patient was decided based on the clinical judgment of paramedics. In general, a case with physical injury caused by external force would be classified as a trauma case. The information is included in this study because it was recorded in the Taipei City Fire Department's database. It is useful when accounting for the possible effects of the popularity of motorcycle use in Taiwan. Information about disease type was not included in this dataset because paramedics are not expected to make diagnoses.

\subsubsection{Meteorological data}

Daily meteorological data were downloaded in time series format from the Central Weather Bureau Observation Data Inquiry System (Central Weather Bureau 2019). From the 16 meteorological stations in the city, the Taipei meteorological station was selected to represent weather conditions in the city because of its location within the Taipei metropolitan area and proximity to sea level. Moreover, the measurements of this meteorological station are relatively complete compared with those of the 15 other stations in the city. The meteorological variables considered in this study include average temperature $\left(T_{\mathrm{avg}}\right)$, minimum temperature ( $\left.T_{\min }\right)$, maximum temperature $\left(T_{\max }\right)$, cloud amount, relative humidity (RH), wind speed (WS), barometric pressure (BP), precipitation, and visibility.

\subsection{Data analysis}

\subsubsection{Data preprocessing}

Before the data analysis, the EAS usage records were aggregated into daily time series data based on their date. In this way, the format of the EAS data was consistent with that of the meteorological data for conducting the data analysis. The 3day moving average smoothing technique was applied to the EAS demand time series data to remove large, unexpected fluctuations resulting from disastrous events. Normalization was not applied to the data series because the yearly EAS demand did not significantly increase within the data period.

\subsubsection{Time-lag analysis}

After completing the above data preprocessing steps, time-lag analysis was carried out to ascertain the time-lag effects of temperature on EAS demand. To achieve this, the EAS demand time series with different days of time lag were regressed on $T_{\text {avg }}$ and average temperature squared $\left(T_{\text {avg }}^{2}\right)$ to find the lag effect of temperature on EAS demand. The number of days of time lag that yielded the regression model with the highest adjusted- $R^{2}$ was considered in the subsequent analysis. In this time-lag analysis, both $T_{\text {avg }}$ and $T_{\text {avg }}{ }^{2}$ were included to address the quadratic relationship between $T_{\text {avg }}$ and EAS demand. Both $T_{\text {avg }}$ and $T_{\text {avg }}{ }^{2}$ were selected because they represent the $U$ shape pattern of the relationship between $T_{\text {avg }}$ and EAS demand. The U shape relationship is applicable to both the summer and winter periods, during which the EAS demand will increase when $T_{\text {avg }}$ increases and decreases respectively. If only the first-order term (i.e., $T_{\text {avg }}$ ) is selected, the regression model formulated will be a straight line, not a $U$ shape model. The U shape model has been observed in previous studies and considered the main focus of the meteorological effects on EAS demand (Lai and Wong 2015; Wong and Lai 2012; Wong et al. 2017; Chan et al. 2011; Iñiguez et al. 2010; Lindeboom et al. 2012; Yu et al. 2019). Moreover, the U shape pattern of the effects of $T_{\text {avg }}$ on EAS demand can easily be understood by a layman. Placing the focus on $T_{\text {avg }}$ can make the final regression model of the relationship between weather and EAS demand easier to interpret from a layman's perspective. 


\subsubsection{Analyzing the effects of weather on EAS demand}

After the time-lag analysis, the EAS demand time series data were split into different groups of data based on the value of the variables in the EAS usage records in order to represent demand from different groups of EAS users. In addition, all the EAS demand time series were shifted forward based on the number of days of time lag identified from the time-lag analysis in order to account for the time-lag effects. Each of the generated EAS demand time series data was then regressed on all weather factors via multivariate forward regression. Forward regression was selected because the algorithm would prioritize the most predominant factors (e.g., $T_{\text {avg }}$ and $T_{\text {avg }}{ }^{2}$ ) in the regression analysis. To be more precise, the forward regression analysis would only include suitable predictors in the regression model based on the forward variable selection method. The forward variable selection method would first assess all the independent variables one by one. The independent variable that satisfied the entry criterion (the significant level of the independent variable's $F$ value $<0.05$ ) and had the highest correlation with the dependent variable would be included in the model. After the first independent variable had been included in the model, the independent variable that satisfied the entry criterion and had the largest partial correlation would be included in the model. This variable selection step would be repeated until none of the unselected independent variables satisfied the entry criterion (IBM SPSS 2017). Linear models with an assumed Poisson distribution were not considered because the distribution of the daily ambulance demand counts was close to normal, and the data was no longer counting values after the 3-day moving average smoothing technique had been applied. Moreover, when using the same analysis method (i.e., multivariate forward regression) adopted in Wong and Lai's (2012) study, the results of the two studies can be more easily compared.

Twelve weather factors were considered, including (1) $T_{\text {avg }}$; (2) $T_{\text {avg }}^{2}$; (3) $T_{\max }-T_{\min }$; (4) $T_{\text {avg }}$ difference between 2 days; (5) $T_{\text {avg }}$ difference among 3 days; (6) RH; (7) RH $\times$ $T_{\text {avg; }}$; (8) WS; (9) BP; (10) precipitation; (11) visibility; and (12) cloud amount. These factors were selected because they are the most common factors that can be calculated from basic meteorological variables. Moreover, they were also adopted in Wong and Lai's (2012) study, so selecting the same set of variables could facilitate result comparison. In addition, the effect of weekends and holidays can also be controlled by including a binary variable in the regression models. Assuming all the factors are included in the model, the model can analytically be written as shown below. In the equation, $B_{1}$ to $B_{13}$ are the regression coefficient of the corresponding independent variable in the model.

$$
\begin{aligned}
\text { EAS demand }= & \text { Constant }+B_{1}\left(T_{\text {avg }}\right)+B_{2}\left(T_{\text {avg }}^{2}\right) \\
& +B_{3}\left(T_{\max }-T_{\min }\right) \\
& +B_{4}\left(T_{\text {avg }} \text { difference between two days }\right) \\
& +B_{5}\left(T_{\text {avg }} \text { difference among three days }\right) \\
& +B_{6}(\mathrm{RH})+B_{7}\left(\mathrm{RH} \times T_{\text {avg }}\right) \\
& +B_{8}(\text { Cloud amount })+B_{9}(\mathrm{BP}) \\
& +B_{10}(\text { Precipitation })+B_{11}(\text { Visiblity }) \\
& +B_{12}(\mathrm{WS})+B_{13}(\text { Weekend and holiday })
\end{aligned}
$$

After comparing the effect sizes calculated using adjusted$R^{2}$ of the regression model of different groups of EAS users, the group of users most sensitive to weather could be identified based on the effect size classification system (Ellis 2010). As the effect size classification system is based on $R\left(\operatorname{not} R^{2}\right)$, the regression models' adjusted- $R^{2}$ were transferred to $R$ before being applied to the system (small: $R<0.3$; medium: $R \geq$ 0.30 and $R<0.5$; large: $R \geq 0.5$ ).

\section{Results}

Table 2 presents the results of the lag effect analysis. In addition to the population as a whole, the elderly (aged 65+ years) were considered independently in the analysis because they were believed to be the most vulnerable group based on previous studies (Wong and Lai 2012). The 4-day time-lag EAS demand data showed the highest correlation with $T_{\text {avg }}$ and $T_{\text {avg }}{ }^{2}$, which obtained adjusted- $R^{2}$ values of 0.15 and 0.28 respectively in the regression model. Therefore, the 4-day time-lag EAS demand data were used in the subsequent analysis.

Table 3 shows the effects of weather factors on daily EAS demand from different age groups. Model (a) only contains temperature-related variables, while model (b) contains all the weather-related variables mentioned in the "Data" section. The adjusted- $R^{2}$ of the regression model (b) on the whole population was 0.16 with a medium-sized effect (Ellis 2010). After analyzing the demands of different age groups, the model for the elderly (aged 65+ years) had the highest adjusted- $R^{2}(0.30)$ with a large effect, while the models for

Table 2 Lag effect analysis of two target age groups based on adjusted$R^{2}$

\begin{tabular}{lcccccc}
\hline Service group & Lag 0 & Lag 1 & Lag 2 & Lag 3 & Lag 4 & Lag 5 \\
\hline All age groups & 0.07 & 0.07 & 0.10 & 0.14 & 0.15 & 0.14 \\
$65+$ & 0.23 & 0.21 & 0.24 & 0.28 & 0.28 & 0.26 \\
\hline
\end{tabular}


Table 3 Results of regression analysis by age groups (4-day time-lag data)

\begin{tabular}{|c|c|c|c|c|c|c|c|c|c|c|}
\hline \multirow[t]{2}{*}{ Weather factor } & \multicolumn{2}{|l|}{ All ages } & \multicolumn{2}{|l|}{$0-14$} & \multicolumn{2}{|l|}{$15-34$} & \multicolumn{2}{|l|}{$35-64$} & \multicolumn{2}{|l|}{$65+$} \\
\hline & (a) & (b) & (a) & (b) & (a) & (b) & (a) & (b) & (a) & (b) \\
\hline Constant & $512.16 * * *$ & $503.76^{* * *}$ & $6.29 * * *$ & $6.29 * * *$ & $77.87 * * *$ & $86.16^{* * *}$ & $114.58 * * *$ & $114.91 * * *$ & $178.26^{* * *}$ & $427.08 * * *$ \\
\hline$T_{\text {avg }}$ & $-11.04 * * *$ & $-10.59 * * *$ & - & - & - & - & - & - & $-5.64 * * *$ & $-5.91 * * *$ \\
\hline$T_{\text {avg }}^{2}$ & $0.21 * * *$ & $0.20 * * *$ & - & - & - & - & - & - & $0.10^{* * * *}$ & $0.10^{* * * *}$ \\
\hline$T_{\max }-T_{\min }$ & - & - & - & - & - & $-0.33^{*}$ & - & - & $0.45 * *$ & $0.44 * *$ \\
\hline $\begin{array}{l}T_{\text {avg }} \text { difference between } \\
2 \text { days }\end{array}$ & - & - & - & - & - & - & - & - & - & - \\
\hline $\begin{array}{l}T_{\text {avg }} \text { difference among } \\
3 \text { days }\end{array}$ & - & - & - & - & $-0.36^{* *}$ & $-0.35^{* *}$ & $-0.42 * *$ & $-0.43 * *$ & $0.35^{*}$ & $0.42 * *$ \\
\hline $\mathrm{RH}$ & & - & & - & & $-0.14 * *$ & & - & & - \\
\hline $\mathrm{RH} \times T_{\text {avg }}$ & & - & & - & & - & & $-0.00 * * *$ & & - \\
\hline Cloud amount & & - & & - & & - & & - & & - \\
\hline BP & & - & & - & & - & & - & & $-0.24 *$ \\
\hline Precipitation & & - & & - & & - & & $0.06^{*}$ & & - \\
\hline Visibility & & $0.55^{*}$ & & - & & $0.24 *$ & & $0.56^{* * *}$ & & - \\
\hline WS & & - & & - & & 0.50 & & - & & - \\
\hline $\begin{array}{l}\text { Weekend and public } \\
\text { holiday }^{(\mathrm{c})}\end{array}$ & -1.62 & -1.54 & $-0.52 * * *$ & $-0.52 * * *$ & -1.09 & -0.85 & 1.54 & $1.66^{*}$ & 0.85 & 0.83 \\
\hline Adjusted- $R^{2}$ & 0.15 & 0.16 & 0.02 & 0.02 & 0.01 & 0.05 & 0.01 & 0.05 & 0.29 & 0.30 \\
\hline
\end{tabular}

the other age groups only had adjusted- $R^{2}(0.02-0.05)$ with a small effect. The effect size difference of the variance explained by the models was considered meaningful, because the 0.25 difference on the adjusted- $R^{2}$ (i.e., $0.3-0.05$ ) can be interpreted as a large-sized effect (Ellis 2010). The result for the elderly (aged 65+ years) shows that BP was negatively related to EAS demand $(B=-0.24 ; p<0.05)$, and model (b)'s adjusted- $R^{2}$ was 0.01 larger than for model (a). Both models (a) and (b) included the control variable representing weekends and public holidays, and none of the models' control variables was significant $(p>0.05)$. Regarding model (b) for the whole population, visibility was positively related to EAS demand $(B=0.55 ; p<0.05)$ and the control variable was insignificant $(p>0.05)$. Including the factor of visibility in the model improved the model adjusted- $R^{2}$ by 0.01 . However, the effect size of the improvements was too low to be considered meaningful.

Table 4 presents the results of the regression analysis by triage level. The patients with triage level 1 were most sensitive to weather (adjusted- $R^{2}=0.23$ ), with a medium-sized effect. Although the models for level 2 and 3 patients also had a medium-sized effect, their adjusted- $R^{2}$ values were relatively smaller than those for level 1 patients (level 1, $0.23 \mathrm{vs}$. levels 2 and 3, 0.09 and 0.09). The effect size difference of the variance explained by the models was considered meaningful, because the 0.14 difference on the adjusted- $R^{2}$ (i.e., $0.23-$ 0.09 ) can be interpreted as a medium-sized effect (Ellis 2010). The effect size of the models for triage levels 4 and 5 can be neglected. When comparing the triage level 1 group's models (a) and (b), three new predictors (i.e., cloud amount, $\mathrm{BP}$, and visibility) were discovered after considering meteorological factors other than temperature. All of them were negatively related to EAS demand (cloud amount: $B=-0.01$, $p<0.05$; BP: $B=-0.08, p<0.05$; visibility: $B=-0.09$, $p<0.05$ ), but the improved model $R^{2}$ was only 0.02 , which is not large enough to be considered meaningful. In both models (a) and (b), the control variable for weekends and public holidays was insignificant $(p>0.05)$.

Table 5 presents the results of the regression analysis by gender and case nature. Female patients were more sensitive to weather than male patients, with a 0.08 difference in adjusted $-R^{2}$ and a small effect size. The effect size difference of the variance explained by the models could not be considered meaningful because the difference can only be interpreted as a small effect (Ellis 2010). Both the female and male groups' model (a) can be improved by including meteorological factors other than temperature. The two sets of variables, (RH: $B=0.063, p<0.001$; $\mathrm{RH} \times T_{\text {avg }}: B=-0.03, p<0.001$; and visibility: $B=0.42$, 
Table 4 Results of regression analysis by triage level (4-day time-lag data)

\begin{tabular}{|c|c|c|c|c|c|c|c|c|c|c|}
\hline \multirow[t]{2}{*}{ Weather factor } & \multicolumn{2}{|l|}{ Level 1} & \multicolumn{2}{|l|}{ Level 2} & \multicolumn{2}{|l|}{ Level 3} & \multicolumn{2}{|l|}{ Level 4} & \multicolumn{2}{|l|}{ Level 5} \\
\hline & (a) & (b) & (a) & (b) & (a) & (b) & (a) & (b) & (a) & (b) \\
\hline Constant & $45.34 * * *$ & $125.00 * *$ & $82.00 * * *$ & $87.21 * * *$ & $156.75 * * *$ & $156.03 * * *$ & $38.32 * * *$ & $30.12 * * *$ & $4.01 * * *$ & $4.01 * * *$ \\
\hline$T_{\text {avg }}$ & $-1.75^{* * *}$ & $-1.99 * * *$ & $-2.50^{* * *}$ & $-2.80^{* * *}$ & $-0.74 * * *$ & - & - & - & $-0.12^{*}$ & $-0.12 *$ \\
\hline$T_{\text {avg }}^{2}$ & $0.03 * * *$ & $0.04 * * *$ & $0.05^{* * *}$ & $0.06^{* * *}$ & - & - & - & - & $0.00 * *$ & $0.00 * *$ \\
\hline$T_{\max }-T_{\min }$ & $0.14 * *$ & $0.24 * * *$ & $0.27 * *$ & $0.36^{* * *}$ & - & - & - & - & - & - \\
\hline$T_{\text {avg }}$ difference between 2 days & - & - & - & - & - & - & - & - & - & - \\
\hline$T_{\text {avg }}$ difference among 3 days & $0.22 * * *$ & $0.27 * * *$ & - & - & - & - & - & - & - & - \\
\hline $\mathrm{RH}$ & & - & & - & & - & & $0.10 * *$ & & - \\
\hline $\mathrm{RH} \times T_{\text {avg }}$ & & - & & - & & $-0.01 * * *$ & & - & & - \\
\hline Cloud amount & & $-0.01^{*}$ & & - & & $-0.08 * *$ & & $0.02 *$ & & - \\
\hline $\mathrm{BP}$ & & $-0.08 *$ & & - & & - & & - & & - \\
\hline Precipitation & & & & - & & $0.09 * *$ & & - & & - \\
\hline Visibility & & $-0.09 *$ & & $-0.35^{* * *}$ & & $0.82 * * *$ & & - & & - \\
\hline WS & & - & & - & & - & & - & & - \\
\hline Weekend and public holiday ${ }^{(\mathrm{c})}$ & 0.05 & 0.01 & 0.07 & 0.01 & 1.75 & 2.08 & -0.21 & -0.28 & $-0.21 * *$ & $-0.21 * *$ \\
\hline Adjusted- $R^{2}$ & 0.21 & 0.23 & 0.06 & 0.09 & 0.05 & 0.09 & 0.00 & 0.01 & 0.02 & 0.02 \\
\hline
\end{tabular}

$* p<0.05 ; * * p<0.01 ; * * * p<0.01$

(a) Only factors of temperature were considered

(b) All factors in the table were considered

(c) The factor of weekend and public holiday was controlled

Table 5 Results of regression analysis by gender and case nature (4-day time-lag data)

\begin{tabular}{|c|c|c|c|c|c|c|c|c|}
\hline \multirow[t]{2}{*}{ Weather factor } & \multicolumn{2}{|l|}{ Female } & \multicolumn{2}{|l|}{ Male } & \multicolumn{2}{|l|}{ Trauma } & \multicolumn{2}{|l|}{ Non-trauma } \\
\hline & (a) & (b) & (a) & (b) & (a) & (b) & (a) & (b) \\
\hline Constant & $161.52 * * *$ & $93.44 * * *$ & $219.92 * * *$ & $191.93 * * *$ & $173.39 * * *$ & $-182.79 *$ & $334.51 * * *$ & $892.43 * * *$ \\
\hline$T_{\text {avg }}$ & $-2.56^{* * *}$ & $1.29 *$ & $-3.22 * * *$ & - & $-0.40 * * *$ & - & $-10.37 * * *$ & $-10.91 * * *$ \\
\hline$T_{\text {avg }}^{2}$ & $0.04 * * *$ & - & $0.06^{* *}$ & - & - & - & $0.21 * * *$ & $0.21 * * *$ \\
\hline$T_{\max }-T_{\min }$ & - & - & - & - & - & - & - & - \\
\hline$T_{\text {avg }}$ difference between 2 days & - & - & - & - & - & - & $0.66^{*}$ & $0.84 * *$ \\
\hline$T_{\text {avg }}$ difference among 3 days & - & - & $-0.45^{*}$ & $-0.66^{* *}$ & $-0.51 * *$ & $-0.53 * *$ & - & - \\
\hline $\mathrm{RH}$ & & $0.63^{* * *}$ & & - & & - & & - \\
\hline $\mathrm{RH} \times T_{\mathrm{avg}}$ & & $-0.03 * * *$ & & $-0.01 * * *$ & & - & & - \\
\hline Cloud amount & & - & & - & & - & & - \\
\hline $\mathrm{BP}$ & & - & & - & & $0.34 * * *$ & & $-0.54 * *$ \\
\hline Precipitation & & - & & $0.08 *$ & & - & & - \\
\hline Visibility & & $0.42 * * *$ & & $0.62 * * *$ & & - & & - \\
\hline WS & & - & & - & & $1.26^{* *}$ & & - \\
\hline Weekend and public holiday ${ }^{(\mathrm{c})}$ & $1.42 *$ & $1.54^{*}$ & -0.70 & -0.23 & $2.27 *$ & $2.52 *$ & $-3.79 * *$ & $-3.86^{* *}$ \\
\hline Adjusted- $R^{2}$ & 0.13 & 0.15 & 0.05 & 0.07 & 0.04 & 0.06 & 0.18 & 0.19 \\
\hline
\end{tabular}

$* p<0.05 ; * * p<0.01 ; * * * p<0.01$

(a) Only factors of temperature were considered

(b) All factors in the table were considered

(c) The factor of weekend and public holiday was controlled 
$p<0.001)$ and $\left(\mathrm{RH} \times T_{\text {avg }}: B=-0.01, p<0.001\right.$; precipitation: $B=0.08, p<0.05$; and visibility: $B=0.62, p<0.001$ ), were found to be the respective predictors of the female and male groups' EAS demand. However, the corresponding improvements in the models after including these factors were only 0.02 with a small effect. On the other hand, the control variable for weekends and public holidays was significant in both models (a) and (b) for the female group $(p<0.05)$.

Meanwhile, the model (a) of non-trauma cases showed a significantly higher adjusted- $R^{2}$ than the corresponding model (a) of trauma cases (trauma 0.04 vs. non-trauma 0.18 ), with a 0.14 difference in adjusted- $R^{2}$ and a medium-sized effect. The effect size difference of the variance explained by the models was considered as meaningful, because the 0.14 difference (i.e., 0.18-0.04) can be interpreted as a medium-sized effect (Ellis 2010). The model (a) for trauma cases can be improved by including meteorological factors other than temperature (i.e., BP: $B=0.34, p<0.001$ and WS: $B=1.26, p<0.01$ ) in the model, and the control variable was significant $(p<0.01)$. Similarly, the model (a) for non-trauma cases can be improved by including the factor of BP $(B=-$ $0.54, p<0.01$ ), and the effect of the control variable was significant $(p<0.01)$. However, the corresponding improvements were only 0.02 and 0.01 respectively; the effects are small.

\section{Discussion}

\subsection{Time-lag effect}

The question of how long people can tolerate adverse weather conditions is related to the time-lag effect. This effect has been observed in other similar studies on mortality (Braga et al. 2002; Leung et al. 2008). The four lag days identified in this study were the same as those recorded in Wong and Lai (2012). Interestingly, the number of lag days identified in studies on mortality was slightly larger than that identified in this study, which focuses on EAS demand (Braga et al. 2002; Leung et al. 2008). Such a small difference may be attributed to the time that patients spend in treatment.

\subsection{Age}

Given their higher vulnerability compared with young people, the elderly were more sensitive to adverse weather conditions than the other populations. However, the sensitivity of infants and young children to weather remains unclear, as they are expected to be less able to protect themselves than adults. Wong and Lai (2012) found that the youngest age group showed the lowest sensitivity to weather, which increased with age. Although this study also found that the elderly were more sensitive to weather, only those patients aged $65+$ years showed sensitivity to weather with a large effect. A linear relationship between sensitivity and age was not observed in this study, but a step function was revealed. Nevertheless, these studies found that infants and young children were insensitive to weather; this may be because they were well cared for by their parents. Following this supposition, future studies conducted in rural and poor areas may find that children left unprotected by their parents are highly sensitive to weather. On the other hand, the extremely crowded living environment in Hong Kong may mean that people aged between 15 and 64 years are highly sensitive to weather compared with the same group in Taipei. Given that the cost of living in Hong Kong is ranked as the secondhighest in the world (Economist Intelligence Unit 2017), many people aged between 15 and 64 years are only able to afford to live in subdivided flats that are around $30 \mathrm{ft}^{2}$ on average (Dwan et al. 2013). These flats do not have windows or air conditioning, and thus have poor air ventilation, which means that their residents suffer during very hot or cold days. On the other hand, in a study in Italy that looked at 5 years' worth of daily emergency ambulance dispatch data, the results showed that EAS demand was higher among the older age group when air temperature was extremely high (Alessandrini et al. 2011).

\subsection{Triage level}

Similarly to the analysis by age, the analysis by triage level showed a step function relationship between sensitivity and triage level. This study, as well as that of Wong and Lai (2012), found that more severe patients were more sensitive to weather. However, the pattern discovered in the latter study was not clear enough because the relationship between weather and triage for level 1 to 3 patients was at a high level. In contrast, the present study showed that the model for triage level 1 patients had a medium-sized effect, while that for triage level 2 and 3 patients had an effect size on the boundary between medium and small. Therefore, the effects of weather on more severe patients were demonstrated much more clearly in this study than in Wong and Lai (2012). In this case, patients were more affected physically than psychologically by adverse weather conditions. If these patients had been mainly affected psychologically, then the triage level 4 and 5 patients would have had models with higher adjusted- $R^{2}$ values because the triage level would have been set according to their physical conditions. Therefore, the result suggests that weather mainly affects patients physically rather than psychologically. Further confirmation of the effects of weather on triage level cases can be carried out if similar research conducted in cities in other parts of the world becomes available in the future. 


\subsection{Gender}

With regard to gender, the results of this study were consistent with those of Wong and Lai (2012). The two studies showed that female patients were relatively more sensitive to weather than male patients, although the effect size differences of the variance explained by the models observed in the two studies were also not large enough to be considered meaningful. Specifically, Wong and Lai (2012) observed 0.04 difference in the adjusted- $R^{2}$ of the models for female and male patients, while the present study observed a larger adjusted- $R^{2}$ difference $(0.08)$, thereby highlighting a gender difference. There have been some similar studies conducted in China that also identified gender differences in the weather's effects on emergency room demand, but the difference was believed to be insignificant (Chen et al. 2015; Zhao et al. 2017). If EAS usage records with more details on case nature can be obtained, then the effects of weather on gynecological conditions can be examined further (Akutagawa et al. 2007; BianchiDemicheli et al. 2001).

\subsection{Case nature}

Unlike Wong and Lai (2012), who did not conduct an analysis by case nature, this study showed that non-trauma cases were more sensitive to weather than trauma cases. This finding may seem inconsistent with that of other studies in Asia that have suggested temperature has a significant effect on trauma cases (Chen et al. 2015; Tai et al. 2007). However, it is possible that the effects of weather on non-trauma cases were stronger than on trauma cases, therefore making the non-trauma patients more sensitive to weather than trauma patients. Given that a significant proportion of Taipei residents are aged 65+ years (over 15\%) and are identified as the most vulnerable group in the city, they greatly contribute to the non-trauma cases. In fact, this population comprised $46.4 \%$ of the non-trauma patients and $19.1 \%$ of the trauma patients in this study. This proportion of non-trauma cases is expected to increase in the future because of Taiwan's aging population (National Development Council 2018). There is also a research study in Korea that has found that the factor of temperature has a larger effect on non-trauma cases than on trauma cases (Kim et al. 2012).

\subsection{Comparison between Taipei and Hong Kong in terms of the effects of weather on EAS demand}

\subsubsection{Similarities}

As discussed in the above subsections, the effects of weather on EAS demand show similar patterns in Taipei and Hong Kong. In general, elderly and critical patients are more sensitive to weather than other populations. The lag effects of temperature on EAS demand in the two cities are also similar. This is expected, because the climatic environments of these cities are relatively similar compared with countries in the equatorial region such as Singapore, which has no cold days. Moreover, given that these two cities are metropolises, most of their residents adopt an urban lifestyle and living environment that differ from those of residents in rural areas. Some differences may also be observed between the two cities because of their different levels of economic development and built environment.

\subsubsection{Differences}

Two major differences were observed between the studies on the effects of weather on EAS demand in Taipei and Hong Kong. First, compared with the Hong Kong study, the Taipei study clearly demonstrated that the elderly and critical patients were most sensitive to weather. This difference can be attributed to the fact that this study directly obtained EAS usage records from the Taipei City Fire Department, while Wong and Lai (2012) only used proxy data from the Hong Kong Hospital Authority, which may have been unable to reflect EAS demand accurately. Given that the Hong Kong Hospital Authority is not the EAS managing authority in Hong Kong, the dataset of Wong and Lai may contain a certain amount of missing cases if the ambulance brought-in indicator was not always filled in appropriately. Moreover, Wong and Lai (2012) omitted EAS calls without patient transfer as well as death before arrival cases, even though these cases only contribute to a small proportion of the total EAS demand. Nevertheless, the macroscopic picture of the results is expected to be correct, and expecting an error-free study would be unrealistic.

Second, Wong and Lai (2012) did not investigate the role of temperature change over the course of a day. They did consider the $T_{\text {avg }}$ difference between two consecutive days in their analysis, but the result of the analysis showed that it was not a significant predictor. Although it remained an insignificant predictor in the Taipei study, the newly added factor in the Taipei study (i.e., temperature change over a day) played an important role for the two most vulnerable groups (i.e., patients aged $65+$ years and triage level 1 patients). This finding is understandable, as temperature change over a day is more difficult to cope with than $T_{\text {avg }}$ change over two consecutive days. People can easily change their clothing on a new day, but may be unable to do so in the middle of the day if they have already left their homes without anticipating a possible change in temperature. If Wong and Lai had considered temperature change over a day in their study, they would likely have found the effects of this variable to be less significant in Hong Kong than in Taipei. This is because temperature change over a day in Taipei is significantly larger in both value 
and variation than that in Hong Kong (Table 1), despite the similarities in their average temperature profiles.

\section{Conclusions}

To conclude, this study has found that the elderly, critical patients, and non-trauma cases are relatively sensitive to weather. The findings of this study are easier to interpret than those of the similar study in Hong Kong (Wong and Lai 2012) because this study used highly accurate EAS usage data and obtained distinct results from the same patient subgroups. This study has also revealed that temperature change over a day, which was not considered in the Hong Kong study, has a significant effect on vulnerable groups.

This study is the first to investigate the effects of weather on daily EAS demand in Taipei. By generating results that are directly applicable to Taipei, this study will prevent the formulation of inappropriate EAS policies. Specifically, the study in Hong Kong showed that EAS policies should target people aged between 35 and 64 years, while the study in Taipei suggested that EAS resources should only focus on the elderly (aged 65 years or above).

After obtaining these preliminary results, a more detailed analysis of different subgroups of patients (e.g., patients with different illnesses, more detailed case natures, and socioeconomic status) should be conducted in order to obtain additional information on when and how paramedics should be equipped to cope with the possible increase in EAS demand in the future. Health education programs should also focus on vulnerable groups in order to increase their awareness and help them protect themselves before the onset of extreme weather conditions, thereby preventing the unnecessary use of EAS.

Acknowledgments We are grateful to the Taipei City Fire Department for access to data records used in the present study.

Authors' contributions HTW, JJL: participated in the design; JJL: data collection; HTW: analysis of data; HTW, JJL: discussion and drafted the manuscript. All of the authors read and approved the final version of the manuscript.

Funding information This work was supported by the grant from the Ministry of Science and Technology, Taiwan (MOST 108-2410-H-110069-MY2).

\section{Compliance with ethical standards}

Ethics approval and consent to participate This study only analyzed anonymous EAS usage records, with no direct or indirect patient contact. The project has certified for exemption from Human Research Ethics Committee at National Cheng Kung University (No. 108-298).

Consent for publication Not applicable.
Competing interests The authors declare that they have no competing interests.

\section{References}

Akutagawa O, Nishi H, Isaka K (2007) Spontaneous delivery is related to barometric pressure. Arch Gynecol Obstet 275:249-254

Alessandrini E, Sajani SZ, Scotto F, Miglio R, Marchesi S, Lauriola P (2011) Emergency ambulance dispatches and apparent temperature: a time series analysis in Emilia-Romagna, Italy. Environ Res 111: $1192-1200$

Bianchi-Demicheli F, Lüdicke F, Spinedi F, Major AL, Kulier R, Campana A, Gyr T (2001) Association between weather conditions and the incidence of emergency gynecological consultations. Gynecol Obstet Investig 51:55-59

Braga AL, Zanobetti A, Schwarz J (2002) The effect of weather on respiratory and cardiovascular deaths in 12 U.S. cities. Environ Health Perspect 110:859-863

Brindle A (2016) A corpus analysis of discursive constructions of the Sunflower Student Movement in the English-language Taiwanese press. Discourse Soc 27:3-19

CCOUC (2019) Climate Change and Health. http://ccouc.org/climatechange-health. Accessed 19 May 2019

Central Weather Bureau (2019) Central Weather Bureau Observation Data Inquire System. http://e-service.cwb.gov.tw/ HistoryDataQuery/index.jsp. Accessed 19 May 2019

Chan EY, Goggins WB, Kim JJ, Griffiths SM (2012) A study of intracity variation of temperature-related mortality and socioeconomic status among the Chinese population of Hong Kong. J Epidemiol Community Health 66:322-327

Chan EY, Goggins WB, Yue JSK, Lee P (2013) Hospital admissions as a function of temperature, weather phenomena and pollution levels in an urban setting in China. Bull World Health Organ 91:576-584

Chan EYY, Goggins WB, Kim JJ, Griffiths S, Ma TKW (2011) Helpseeking behavior during elevated temperature in Chinese population. J Urban Health 88:637

Chapman S, Watson JE, Salazar A, Thatcher M, McAlpine CA (2017) The impact of urbanization and climate change on urban temperatures: a systematic review. Landsc Ecol 32:1921-1935

Chen $Y$ et al (2015) Impact of subtropical climate on frequency of ambulance use for trauma patients in a coastal area of China. Chin J Traumatol 18:141-146

Clarivate Analytics (2018) Journal Citation Reports. https://clarivate. com/products/journal-citation-reports/. Accessed 19 May 2019

Dwan D, Sawicki M, Wong J (2013) Subdivided housing issues of Hong Kong: causes and solutions. web.wpi.edu/Pubs/E-project/Available/ E-project-022713-213142/unrestricted/Subdivided Housing Issues_of_Hong_Kong_Causes_and_Solutions.pdf. Accessed 19 May 2019

Economist Intelligence Unit (2017) Worldwide Cost of Living Report 2017. https://www.eiu.com/public/topical_report.aspx? campaignid=WCOL2017. Accessed 19 May 2019

Ellis PD (2010) The essential guide to effect sizes: statistical power, metaanalysis, and the interpretation of research results. Cambridge University Press $173 \mathrm{pp}$

Goggins WB, Chan EY (2017) A study of the short-term associations between hospital admissions and mortality from heart failure and meteorological variables in Hong Kong: weather and heart failure in Hong Kong. Int J Cardiol 228:537-542

Goggins WB, Woo J, Ho S, Chan EY, Chau PH (2012) Weather, season, and daily stroke admissions in Hong Kong. Int J Biometeorol 56: 865-872

Goggins WB, Ren C, Ng E, Yang CY, Chan EY (2013) Effect modification of the association between meteorological variables and 
mortality by urban climatic conditions in the tropical city of Kaohsiung, Taiwan. Geospat Health 8:37-44

Griffiths GM, Chambers LE, Haylock MR, Manton MJ, Nicholls N, Baek HJ, Choi Y, Della-Marta PM, Gosai A, Iga N, Lata R, Laurent V, Maitrepierre L, Nakamigawa H, Ouprasitwong N, Solofa D, Tahani L, Thuy DT, Tibig L, Trewin B, Vediapan K, Zhai P (2005) Change in mean temperature as a predictor of extreme temperature change in the Asia-Pacific region. Int J Climatol 25:1301-1330

IBM SPSS (2017) IBM SPSS statistics base 25. SPSS, Chicago

Iñiguez C, Ballester F, Ferrandiz J, Pérez-Hoyos S, Sáez M, López A (2010) Relation between temperature and mortality in thirteen Spanish cities. Int J Environ Res Public Health 7:3196-3210

Kim Y, Kim H, Shin SD, Hong YC (2012) Different influence of outdoor temperature on traumatic and nontraumatic injuries. J Trauma Acute Care Surg 73:944-949

Lai PC, Wong HT (2015) Weather and age-gender effects on the projection of future emergency ambulance demand in Hong Kong. Asia Pac J Public Health 27:NP2542-NP2554

Lam HC, Li AM, Chan EY, Goggins WB (2016) The short-term association between asthma hospitalisations, ambient temperature, other meteorological factors and air pollutants in Hong Kong: a timeseries study. Thorax. 71:1097-1109

Leung YK, Yip KM, Yeung KH (2008) Relationship between thermal index and mortality in Hong Kong. Meteorol Appl 15:399-409

Lin TK (2015) Influencing factors of emergency medical service demand: an empirical study of New Taipei City. M.Sc. thesis, Dept. of Geography, National Taiwan University

Lindeboom W, Alam N, Begum D, Streatfield PK (2012) The association of meteorological factors and mortality in rural Bangladesh, 19832009. Glob Health Action 5:61-73

Lowthian JA, Cameron PA, Stoelwinder JU, Curtis A, Currell A, Cooke MW, McNeil JJ (2011) Increasing utilisation of emergency ambulances. Aust Health Rev 35:63-69

Mastercard (2017) Mastercard Asia Pacific Destinations Index 2017. http://newsroom.mastercard.com/asia-pacific/files/2017/04/ReportMastercard-Asia-Pacific-Destinations-Index-2017.pdf. Accessed 19 May 2019

National Development Council (2018) Population Projections for R.O.C. (Taiwan): 2018 2065. https://www.ndc.gov.tw/en/cp.aspx?n= 2E5DCB04C64512CC\&s=002ABF0E676F4DB5. Accessed 19 May 2019

Onozuka D, Hagihara A (2016) Spatial and temporal variation in emergency transport during periods of extreme heat in Japan: a nationwide study. Sci Total Environ 544:220-229
Onozuka D, Hagihara A (2017) Spatiotemporal variations of extreme low temperature for emergency transport: a nationwide observational study. Int J Biometeorol 61:1081-1094

Qiu H, Yu ITS, Tse LA, Chan EYY, Wong TW, Tian LW (2015) Greater temperature variation within a day associated with increased emergency hospital admissions for asthma. Sci Total Environ 505:508513

Tai CC, Lee CC, Shih CL, Chen SC (2007) Effects of ambient temperature on volume, specialty composition and triage levels of emergency department visits. Emerg Med J 24:641-644

Thornes JE, Fisher PA, Rayment-Bishop T, Smith C (2014) Ambulance call-outs and response times in Birmingham and the impact of extreme weather and climate change. Emerg Med J 31:220-228

Turner LR, Connell D, Tong S (2013) The effect of heat waves on ambulance attendances in Brisbane, Australia. Prehosp Disaster Med 28:482-487

Vencloviene J, Babarskiene R, Dobozinskas P, Siurkaite V (2015) Effects of weather conditions on emergency ambulance calls for acute coronary syndromes. Int J Biometeorol 59:1083-1093

Wong HT, Lai PC (2012) Weather inference and daily demand for emergency ambulance services. Emerg Med J 29:60-64

Wong HT, Lai PC (2014) Weather factors in the short-term forecasting of daily ambulance calls. Int J Biometeorol 58:669-678

Wong HT, Yin Q, Guo YQ, Murray K, Zhou DH, Slade D (2015) Big data as a new approach in emergency medicine research. J Acute Dis 4: $178-179$

Wong HT, Chiang VCL, Choi KS, Loke AY (2016) The need for a definition of big data for nursing science: a case study of disaster preparedness. Int J Environ Res Public Health 13:1015

Wong HT, Lai PC, Chen S (2017) Biometeorological modelling and forecasting of monthly ambulance demand for Hong Kong. Hong Kong J Emerg Med 24:3

Yang C et al (2014) Acute effect of ambient air pollution on heart failure in Guangzhou, China. Int J Cardiol 177:436-441

Yu X, Lei X, Wang M (2019) Temperature effects on mortality and household adaptation: evidence from China. J Environ Econ Manag 96:195-212

Zhao Q et al (2017) Ambient temperature and emergency department visits: time-series analysis in 12 Chinese cities. Environ Pollut 224:310-316

Publisher's note Springer Nature remains neutral with regard to jurisdictional claims in published maps and institutional affiliations. 